\title{
Cabozantinib as first-line treatment in advanced renal cell carcinoma: a profile of its use
}

\author{
Katherine A. Lyseng-Williamson ${ }^{1}$
}

Published online: 7 August 2018

() Springer Nature 2018, corrected publication 2019

\begin{abstract}
Oral cabozantinib tablets (Cabometyx ${ }^{\circledR}$ ) are an important option for the treatment of advanced renal cell carcinoma (RCC). Cabozantinib is an anti-angiogenic agent and potently inhibits multiple tyrosine kinases, including those implicated in the development of RCC. The previously approved indication of cabozantinib tablets (i.e. treatment of advanced RCC following prior VEGF-targeted therapy) has been extended to include the first-line treatment of advanced RCC in treatment-naïve adults with intermediate or poor risk (EU) and all patients with advanced RCC (USA). These label extensions are based on the results of a randomized, open-label phase 2 trial, in which adults with metastatic RCC of poor or intermediate risk received targeted first-line treatment with cabozantinib or standard-of-care sunitinib. Relative to sunitinib, cabozantinib significantly prolonged median progression-free survival (primary endpoint; investigator and independent assessments), and increased the objective response rate (investigator assessment). The tolerability profile of cabozantinib is comparable to those of other tyrosine kinase inhibitors, with adverse events being manageable with medical intervention, dosage reductions, treatment interruption and/or permanent discontinuation.
\end{abstract}

Adis evaluation of cabozantinib in the first-line

treatment advanced renal cell carcinoma (RCC)

Potent inhibitor of multiple tyrosine kinases implicated in RCC

Significantly prolongs progression-free survival relative to sunitinib in adults with metastatic RCC of poor or intermediate risk

Also improves objective response rates compared with sunitinib in this patient population

Has a manageable tolerability profile
Katherine A. Lyseng-Williamson

dtp@adis.com

1 Springer, Private Bag 65901, Mairangi Bay, Auckland 0754, New Zealand

\section{What is the rationale for using cabozantinib as first-line treatment for advanced renal cell carcinoma (aRCC)?}

Renal cell carcinoma (RCC) is the most common type of kidney cancer (accounts for $\approx 90 \%$ of cases), with most $(\approx 80 \%)$ being of clear-cell histology [1]. At the time of diagnosis, $\approx 20-30 \%$ of patients present with metastatic RCC (mRCC), which has a poor long-term prognosis [1-4]. The development of RCC has been linked to the activation of several receptor tyrosine kinase signalling pathways [e.g. those involving vascular endothelial growth factor (VEGF) receptors (VEGFR), MET, AXL and mTOR], which is often a consequence of the functional disruption of the von Hippel-Lindau tumor suppressor protein [2-4].

Tyrosine kinase inhibitors are widely used in the treatment of advanced RCC (aRCC) [1, 4-6]. One such agent is cabozantinib, with the tablet formulation (Cabometyx ${ }^{\circledR}$ ) $[7,8]$ being initially approved to treat aRCC following prior VEGF-targeted therapy. The initial indication for cabozantinib tablets has recently been extended to include the firstline treatment of aRCC in adults with intermediate or poor risk in the EU [7] and all patients with aRCC in the USA [8]. This review focuses on the use of cabozantinib tablets in the first-line treatment of aRCC, with Table 1 providing a summary of the prescribing information in this setting $[7,8]$. 
Importantly, cabozantinib tablets $[7,8]$ and cabozantinib capsules (Cometriq ${ }^{\circledR}$; approved to treat progressive metastatic medullary thyroid cancer) $[9,10]$ are not bioequivalent [11] and, therefore, cannot be used interchangeably [7, 8]. Consult local information for further details regarding the use of cabozantinib tablets and capsules.

\section{What are the pharmacological properties of cabozantinib?}

\section{Pharmacodynamic properties}

Cabozantinib is a small molecule anti-angiogenic agent and potent targeted inhibitor of multiple tyrosine kinases implicated in the development of RCC (i.e. VEGFR-2, MET and AXL), as well as many others (VEGFR-1 and -3, RET, KIT, FLT3, ROS1, MER, TYRO3, FLT3, TRKB and TIE2) $[3,4,7,8,12-15]$. These tyrosine kinases are associated with tumour angiogenesis and growth, pathological bone remodelling, drug resistance and metastatic progression $[3,7,8,12-15]$. In in vitro studies, the migration, invasion and proliferation of tumour cell lines, including clear-cell RCC lines, were inhibited by cabozantinib [12, 13, 15]. In preclinical tumour models, cabozantinib dose-dependently inhibited the phosphorylation of VEGFR-2 and MET, disrupted tumour vasculature, induced tumour cell apoptosis and did not promote the metastasis of tumours $[12,15]$.

A population exposure-response analysis of cabozantinib in the typical individual with aRCC who had received prior VEGF-targeted therapy was conducted based on data from the METEOR trial [16]. The analysis predicted that exposure to cabozantinib provided by the once-daily $60 \mathrm{mg}$ dosage (i.e. the recommended daily dosage for most patients) would provide greater anti-tumour activity than that provided by once-daily 20 or $40 \mathrm{mg}$ in the typical individual undergoing cabozantinib treatment [16]. However, in some non-typical patients (e.g. those with hepatic impairment or who are concomitantly taking certain other medications), exposure to cabozantinib following administration of once-daily cabozantinib $60 \mathrm{mg}$ may be higher than shown in typical patients, and a reduced dosage of 20 or $40 \mathrm{mg} /$ day may achieve more typical exposure and, as a result, comparable anti-tumour activity (Table 1) [7, 8].

\section{Pharmacokinetic properties}

In healthy adults [11], single doses of cabozantinib 20, 40 and $60 \mathrm{mg}$ tablets showed dose-proportional increases in mean maximum plasma concentrations $\left(\mathrm{C}_{\max }\right)$ of 117,239 and $343 \mathrm{mg} / \mathrm{mL}$, respectively; corresponding median times to $\mathrm{C}_{\max }$ were 3,3 and $4 \mathrm{~h}$ (range 1-120). Based on the area under the plasma time-concentration curve (AUC), cabozantinib accumulates with repeated daily administration (mean accumulation ratio of $\approx 4-5$ for day 19 vs day 1 ), and reaches steady state at $\approx 15$ days $[7,8,15]$.

Cabozantinib has a volume of distribution of $\approx 319 \mathrm{~L}$, is highly ( $\geq 99.7 \%$ ) bound to plasma proteins, has a plasma clearance rate of $2.2 \mathrm{~L} / \mathrm{h}$ at steady state, and has a terminal elimination half-life of $\approx 99 \mathrm{~h}[7,8,15,17]$. Cabozantinib undergoes extensive metabolism, with its four major metabolites comprising $>65 \%$ of the total exposure to the drug [18]; the potency of targeted kinase inhibition of these metabolites is $\leq 10 \%$ of that of the parent drug. It is excreted in the faeces $(\approx 54 \% ; 43 \%$ as the parent drug) and urine $(\approx 27 \%)[7,8,18]$.

Cabozantinib tablets are not bioequivalent to cabozantinib capsules and are not interchangeable [11]. In healthy adults [11], the AUC with cabozantinib tablets differed by $<10 \%$ versus that with capsules (indicating bioequivalence); however, $\mathrm{C}_{\max }$ values were $19 \%$ higher with the tablet relative to the capsule and bioequivalence was not established [11].

Although data are limited, exposure to cabozantinib appears to be somewhat increased in patients with renal and hepatic impairment [17], and appropriate precautions should be followed (Table 1).

\section{Potential food and drug interactions}

As food increases exposure to cabozantinib [19], it should not be taken with food (patients should not eat for $\geq 2 \mathrm{~h}$ before and $\geq 1 \mathrm{~h}$ after administration; Table 1) [7, 8].

Clinically significant pharmacokinetic drug interactions may occur between cabozantinib, a cytochrome P450 (CYP) 3A4 substrate, and strong CYP3A4 inducers and inhibitors (Table 1) $[7,8,15,20]$. Interactions may also occur between cabozantinib and multidrug resistance-associated protein 2 (MRP2) inhibitors, oral contraceptives, P-glycoprotein substrates and warfarin (Table 1) [7, 15]. Appropriate precautions should be followed to ameliorate the risk of clinically relevant drug interactions $[7,8]$.

The concomitant use of cabozantinib and gastric $\mathrm{pH}$ modifying agents (e.g. proton pump inhibitors, histamine $\mathrm{H}_{2}$-receptor antagonists and antacids) does not require dosage adjustment [7, 8], as such agents do not affect cabozantinib exposure [19]. Cabozantinib does not inhibit CYP enzymes and, therefore, is not expected to markedly affect the pharmacokinetic profiles of drugs that undergo CYP metabolism [20].

\section{What is the efficacy of cabozantinib as first-line treatment in aRCC?}

\section{CABOSUN trial}

Cabozantinib provided clinical benefits relative to standard-of-care treatment with sunitinib as initial targeted therapy for adults with clear-cell $\mathrm{mRCC}$ of poor or 
Table 1 Summary of the approved indications and administration of oral cabozantinib tablets (Cabometyx $\left.{ }^{\circledR}\right)$ as first-line treatment for advanced renal cell carcinoma in the EU [7] and USA [8]. Unless otherwise indicated, information applies to both the EU and USA

What are the approved indications of cabozantinib tablets as first-line treatment of RCC?

EU: treatment of advanced RCC in treatment-naïve adults with intermediate or poor risk

USA: treatment of pts with advanced RCC

How is cabozantinib available?

Film-coated tablets containing cabozantinib $(S)$-malate equivalent to 20,40 or $60 \mathrm{mg}$ of cabozantinib

How should cabozantinib be administered?

Administration

Do not eat at least $2 \mathrm{~h}$ before and $1 \mathrm{~h}$ after administration; swallow tablets whole (do not crush)

Duration of treatment

Dosage adjustment

Until the pt is no longer clinically benefitting from therapy or until unacceptable toxicity occurs

Dosage may need to be $\downarrow$ (i.e. from $60 \mathrm{mg}$ /day to $40 \mathrm{mg} /$ day, then to $20 \mathrm{mg} /$ day), or treatment temporarily or permanently discontinued, if serious or unacceptable ADRs occur

How should cabozantinib be used in special populations?

Paediatric pts (aged $\leq 18$ years)

Elderly pts (age $\geq 65$ years)

Efficacy and safety have not been established (no available data)

Pts with renal impairment

No specific dose adjustment is recommended

Mild or moderate impairment: use with caution (EU); no dosage adjustment required (USA)

Severe impairment: use is not recommended (EU); no clinical experience (USA)

Pts with hepatic impairment

Mild or moderate impairment: $\downarrow$ dosage $[40 \mathrm{mg}$ once daily (EU)] due to $\uparrow$ in cabozantinib exposure

Severe impairment: use is not recommended (lack of data)

Patients with cardiac impairment

No specific dose adjustment is recommended (limited data)

What precautions should be taken with cabozantinib with regard to fertility, pregnancy and breast-feeding?

Fertility

EU: advise male and female pts to seek advice and consider fertility preservation prior to starting treatment (based on non-clinical safety findings; no data on human fertility)

Contraception in males and women of child-bearing $\quad$ Female pts and partners of male pts should avoid pregnancy during treatment (EU); advise female pts of potential

risk (USA) [fetal harm has been shown in animals; potential risk in human is unknown]

Female/male pts and their partners (EU) or female pts (USA) should use effective methods of contraception during treatment and for $\geq 4$ months after completing treatment

Pregnancy

Use only if the clinical condition requires cabozantinib treatment (EU); advise pts of risk (USA)

Breast-feeding

Discontinue breast-feeding during treatment and for $\geq 4$ months after completing treatment (no known whether cabozantinib and its metabolites are excreted into breast milk)

What clinically relevant drug and food interactions may potentially occur with cabozantinib?

\begin{tabular}{|c|c|}
\hline Strong CYP3A4 inducers ${ }^{\mathrm{a}}$ & $\begin{array}{l}\text { Cabozantinib exposure may } \downarrow \text {; avoid chronic concomitant use (EU); } \uparrow \text { cabozantinib dosage if concomitant } \\
\text { use cannot be avoided (USA) }\end{array}$ \\
\hline Strong CYP3A4 inhibitors ${ }^{\mathrm{a}}$ & $\begin{array}{l}\text { Cabozantinib exposure may } \uparrow \text {; use concomitantly with caution (EU); } \downarrow \text { cabozantinib dosage if concomi- } \\
\text { tant use cannot be avoided (USA) }\end{array}$ \\
\hline MRP2 inhibitors ${ }^{\mathrm{a}}$ & EU: use concomitantly with caution (cabozantinib exposure may $\uparrow$ ) \\
\hline OCs & EU: use an additional contraceptive method (effect of OC may $\downarrow$; lack of PK studies) \\
\hline P-gp substrates ${ }^{\mathrm{a}}$ & EU: use concomitantly with caution (exposure to P-gp substrates may $\uparrow$ ) \\
\hline Warfarin & EU: monitor INR values (risk of plasma protein displacement of warfarin) \\
\hline
\end{tabular}

$C Y P$ cytochrome $\mathrm{P} 450, I N R$ international normalized ratio, $M R P 2$ multidrug resistance-associated protein $2, O C$ oral contraceptives, $P$ - $g p$ P-glycoprotein substrates, $P K$ pharmacokinetic, $p t$ patient, $R C C$ renal cell carcinoma, $\uparrow$ increase, $\downarrow$ decrease

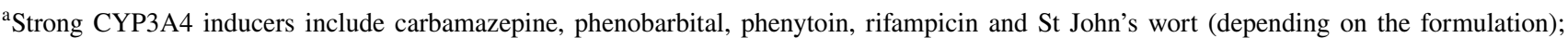
strong CYP3A4 inhibitors include clarithromycin, erythromycin, itraconazole, ritonavir and grapefruit juice; MRP2 inhibitors include ciclosporin, efavirenz and emtricitabine; P-gp substrates include aliskiren, ambrisentan, colchicine, dabagatran etexilate, digoxin, fexofenadine, maraviroc, posaconazole, ranolazine, saxagliptin, sitagliptin, talinolol and tolvaptan

intermediate risk in the open-label, multicentre phase 2 CABOSUN trial [21]. Prior to randomization, patients were stratified by their International Metastatic Renal Cell Carcinoma Database Consortium (IMDC) risk (80.9 and $19.1 \%$ of patients were in the IMDC intermediateand poor-risk groups, respectively) and presence of bone metastases (36\% of patients had bone metastases).

Over the 24-month enrolment period (July 2013 to 6 April 2015, patients were randomized to receive [21]:
- Cabozantinib $60 \mathrm{mg}$ once daily every day of each 6 -week cycle [intent-to-treat (ITT) $n=79$ ]. Dosage reductions to 40 and $20 \mathrm{mg}$ once daily were permitted if required for tolerability-related issues.

- Sunitinib $50 \mathrm{mg}$ once daily for the first 4 weeks of each 6-week cycle, followed by a 2 -week break (ITT $n=78$ ). Dosage reductions to 37.5 and $25 \mathrm{mg}$ once daily were permitted if required for tolerability-related issues. 
The 6-week treatment cycles were continued until disease progression, therapy intolerance or consent withdrawal [21]. The primary endpoint was progression-free survival (PFS) in the ITT population, with the final analysis being conducted when 123 PFS events (progression or death) had occurred (11 April 2016). Prior to the data cut-off, treatment was discontinued by $65 / 78(83 \%)$ and $70 / 72(99 \%)$ of patients who received at least one dose of cabozantinib or sunitinib. Of those patients, disease progression was the most common reason for discontinuing treatment (65 and $57 \%$ of patients who discontinued cabozantinib and sunitinib, respectively), followed by treatment-emergent adverse events (TEAEs) [25 and 23\%] [21].

Median PFS was significantly longer with cabozantinib than with sunitinib in the treatment of $\mathrm{mRCC}$ in patients with poor or intermediate risk $[21,22]$. Relative to sunitinib, cabozantinib reduced the risk of disease progression or death by $34 \%$ based on data up to 11 April 2016 in the investigator assessment [21], and by 44 and $52 \%$ based on data as of 15 September 2016, in the investigator and independent assessments, respectively [22] (Table 2). In subgroup analyses by stratification factors based on 11 April 2016 data, median PFS was longer with cabozantinib than with sunitinib in the IMDC intermediate-risk and bone-metastases subgroups, but not in the other subgroups (Table 2) [21]. In contrast, in the independent assessment based on data as of 15 September 2016, median PFS was longer with cabozantinib than with sunitinib in the subgroups of patients with IMDC intermediate or poor risk, with and without bone metastases, and who were MET positive, but not those who were MET negative (Table 2) [22].

Overall response rates (i.e. proportion of patients with a confirmed complete or partial response) were higher with cabozantinib than with sunitinib [33\% (95\% CI 23-44) vs $12 \%$ (95\% CI 5.4-21)] based on data as of 11 April 2016 [21], but not in the independent review of 15 September 2016 data [20\% (95\% CI 12-31) vs 9\% (95\% CI 4-18)] [22]. Confirmed partial responses comprised most of the overall responses (31.6 and $11.5 \%$ of cabozantinib and sunitinib recipients in the earlier investigator assessment [21] and all patients in the later independent assessment [22]). As of 11 April 2016, 46 and 42\% of cabozantinib and sunitinib recipients, respectively had stable disease, and 18 and $26 \%$ had progressive disease [21]; corresponding results as of 15 September 2016 were 54 and $38 \%$ of patients with stable disease, and 18 and $29 \%$ with progressive disease [22]. Data were missing or not evaluable for 3.8 and $20.5 \%$ of patients in the cabozantinib and sunitinib groups, respectively, as of 11 April 2016 [21], and 8 and $23 \%$ of patients as of 15 September 2016 [22].

Although CABOSUN was not powered to detect betweengroup differences in overall survival (OS), median OS was numerically, but not statistically, longer with cabozantinib than with sunitinib at the data cut-off date of 15 September 2016 [21] and at the updated cut-off date of 1 July 2017 [22] (Table 2).

Cabozantinib may be more effective than sunitinib with regard to other secondary endpoints (data available from abstracts) [23, 24]. Eastern Cooperative Oncology Group performance status (ECOG-PS) scores were used a proxy for assessing health-related quality-of-life [23]. As expected, ECOG-PS scores were better before disease progression than after disease progression (e.g. across evaluable patients in both treatment groups, $40 \%$ had an ECOG-PS score of 0 pre-progression vs $25 \%$ post-progression; mean ECOG-PS scores were 0.69 pre-progression vs 0.91 post-progression). The risk of deterioration in performance (i.e. decreases in ECOG-PS scores from 0 to 2, 1-3, or 2-3) was numerically, but not statistically, lower with cabozantinib than with sunitinib (HR 0.44; 95\% CI 0.16-1.26) [23].

The quality-adjusted time without symptoms or toxicity (Q-TWiST) was longer with cabozantinib than with sunitinib [24]. The analysis was based on data for 650 days (i.e. the median survival follow-up period), divided the OS of each patient into three health states, and weighted each state with a health utility of 0 (death) to 1 perfect health). With regards to the mean duration in each of the three health states, relative to sunitinib, cabozantinib had an 8-day longer time with grade 3/4 toxicity pre-progression, a 121-day longer time without symptoms of disease progression or grade 3/4 toxicity, and a 104-day shorter time after progression or relapse. The duration of Q-TWiST, which takes into account the disease control and toxicity provided by each treatment and the HR-QOL of each health state, was 17-129 days longer with cabozantinib than with sunitinib, depending on the utility values incorporated [24].

\section{Meta-analyses}

Recent network meta-analyses (NMAs) [25, 26] have evaluated the efficacy of first-line treatments for $\mathrm{mRCC} / \mathrm{aRCC}$. One NMA investigated cabozantinib versus standard-ofcare options in the first-line treatment of $\mathrm{mRCC} / \mathrm{aRCC}$ based on IMDC risk [25]. In intermediate-risk patients ( $n=64-466)$, the use of cabozantinib with was significantly favoured over that of sunitinib (HR $0.52 ; 95 \% \mathrm{CI}$ $0.33-0.82)$, sorafenib $(0.46 ; 0.26-0.80)$, interferon (IFN; $0.20 ; 0.12-0.36)$, and bevacizumab + IFN (0.37 0.20-0.68) with regard to PFS. Similar PFS results were shown in poor-risk patients $(n=15-257)$, with the use of cabozantinib being significantly favoured over that of sunitinib (HR 0.31; 95\% CI 0.11-0.90), temsirolimus (0.22; 0.06-0.87), IFN (0.16; 0.04-0.64), and bevacizumab + IFN (0.20; 0.05-0.88). With regard to OS, HRs favoured of cabozantinib over each comparator [HR 0.63-0.89 in intermediate-risk $(n=63-403)$ and $0.34-0.60$ in poor-risk 
Table 2 Efficacy of cabozantinib versus sunitinib as initial targeted therapy for patients with metastatic renal cell carcinoma of poor or intermediate risk in the randomized, open-label phase 2 CABOSUN trial $[21,22]$

Endpoint (data cut-off date)

Median no. of months $(95 \% \mathrm{CI})$

Cabozantinib vs sunitinib HR $(95 \% \mathrm{CI})$ Cabozantinib $(n=79) \quad$ Sunitinib $(n=78)$

\section{Median PFS}

\begin{tabular}{|c|c|c|c|}
\hline PFS in total population $\left(11\right.$ April 2016) ${ }^{\mathrm{a}}[21]$ & $8.2(6.2-8.8)$ & $5.6(3.4-8.1)$ & aHR (P or D): $0.66(0.46-0.95)^{*}$ \\
\hline PFS in total population (15 September 2016) [22] & $8.3(6.5-12.4)$ & $5.4(3.4-8.2)$ & HR (P or D): $0.56(0.37-0.83)^{* *}$ \\
\hline $\begin{array}{l}\text { Independent review of PFS in total population (15 } \\
\text { September 2016) [22] }\end{array}$ & $8.6(6.8-14.0)$ & $5.3(3.0-8.2)$ & HR (P or D): $0.48(0.31-0.74)^{* * *}$ \\
\hline \multicolumn{4}{|l|}{ PFS in pt subgroups ${ }^{b}$ (11 April 2016) [21] } \\
\hline IMDC intermediate risk & 8.3 & 6.2 & HR (P or D): $0.64(0.43-0.96)^{c}$ \\
\hline IMDC poor risk & 6.1 & 2.8 & HR (P or D): 0.75 (0.35- 1.65) \\
\hline Bone metastases present & 6.1 & 3.4 & HR (P or D): $0.54(0.31-0.95)^{\mathrm{c}}$ \\
\hline Bone metastases absent & 8.6 & 7.6 & HR (P or D): $0.78(0.48-1.21)$ \\
\hline \multicolumn{4}{|l|}{ PFS in pt subgroups ${ }^{b}$ (15 September 2016) [22] } \\
\hline IMDC intermediate risk & 11.4 & 6.1 & HR (P or D): $0.52(0.32-0.82)^{c}$ \\
\hline IMDC poor risk & 6.8 & 2.7 & HR (or D): $0.31(0.11-0.92)^{\mathrm{c}}$ \\
\hline Bone metastases present & 5.5 & 3.3 & HR (P or D): $0.51(0.26-0.99)^{c}$ \\
\hline Bone metastases absent & 11.4 & 5.7 & HR (P or D): $0.50(0.29-0.85)^{\mathrm{c}}$ \\
\hline MET positive & 13.8 & 3.0 & HR (P or D): $0.32(0.16-0.63)^{\mathrm{c}}$ \\
\hline MET negative & 6.9 & 6.1 & HR (P or D): $0.67(0.37-1.23)$ \\
\hline \multicolumn{4}{|l|}{ Median OS } \\
\hline OS (15 September 2016) [21] & $30.3(14.6-35.0)$ & $21.8(16.3-27.0)$ & aHR (D): 0.80 (0.50-1.26) \\
\hline Updated OS (1 July 2017) [22] & 26.6 (14.6 to NE) & $21.2(16.3-27.4)$ & aHR (D): $0.80(0.53-1.21)$ \\
\hline
\end{tabular}

Pts received cabozantinib $60 \mathrm{mg}$ once daily every day of each 6-week cycle or sunitinib $50 \mathrm{mg}$ once daily for the first 4 weeks of each 6-week cycle, followed by a 2-week break (dosage reductions to cabozantinib 40 and $20 \mathrm{mg} /$ day or sunitinib 37.5 and $25 \mathrm{mg} /$ day were permitted for tolerability issues). Unless otherwise indicated, results presented are in the intent-to-treat population and were assessed by investigators. KaplanMeier analyses were used to estimate OS and PFS distributions by treatment arm

$a H R$ adjusted HR, $D$ death, $H R$ hazard ratio, IMDC International Metastatic Renal Cell Carcinoma Database Consortium, $N E$ not estimable, $O S$ overall survival, $P$ disease progression, $P F S$ progression-free survival, $p t$ patient

$* p=0.012, * * p=0.0042, * * * p=0.0008$ vs sunitinib

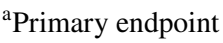

${ }^{\mathrm{b}}$ At baseline in the subgroups, 127 pts were of IMDC intermediate risk (64 with cabozantinib and 63 with sunitinib), 30 were of IMDC poor risk (15 and 15), 57 had bone metastases present (29 and 28), 100 did not have bone metastases (50 and 50), 62 were MET positive (32 and 30;) and 69 were MET negative (39 and 30) [MET status data missing for $26 \mathrm{pts}$ ]

${ }^{\mathrm{c}} 95 \%$ CIs indicate that cabozantinib is favoured over sunitinib

patients $(n=15-257)]$, but did not achieve statistical significance.

The other NMA compared cabozantinib with other firstline systemic treatment of mRCC [26]. Based on the PFS results of 10 trials in 4819 patients, there was a $91 \%$ probability that cabozantinib was the preferred first-line treatment. The use of cabozantinib was significantly favoured over that of atezolizumab, atezolizumab + bevacizumab, nivolumab + ipilimumab, pazopanib, sorafenib and sunitinib, with these drugs having a 1.8- to 2.3-fold higher risk of PFS. Although axitinib, pazopanib + everolimus and tivozanib had a 1.7- to 1.8 -fold higher risk of PFS than cabozantinib, the $95 \%$ CIs for these HRs indicated that the values were non-significant. Based on OS results in 5 trials in 3379 patients, cabozantinib was favoured over sunitinib (HR 0.80; 95\% CI 0.53-1.2), but not nivolumab + ipilimumab (1.2; 0.73-1.9), which has a $48 \%$ probability of being the preferred first-line treatment.

\section{What is the tolerability profile of cabozantinib in aRCC?}

Cabozantinib has a generally manageable tolerability profile when used as the first-line treatment of aRCC, with an overall profile comparable to those of other tyrosine kinase inhibitors in this setting [21,27]. Treatment-related adverse events (TRAEs) associated with cabozantinib can generally be linked to its inhibition of receptor tyrosine kinases, such as VEGFR2, KIT and other targets [27]. 
In the CABOSUN trial in patients with clear-cell mRCC of poor or intermediate risk, TEAEs with first-line treatment with cabozantinib and sunitinib were consistent with the known tolerability profiles of these drugs [21]. TEAE severity was graded according to National Cancer Institute Common Terminology Criteria for Adverse Events version 4.0. At the time of the descriptive tolerability analysis in the safety population, the median duration of treatment was 6.9 months (range 0-26.2) in 78 cabozantinib recipients, and 2.8 months (range 0-23.5) in 72 sunitinib recipients [21].

The incidence of grade 1-2 and grade 3-4 TEAEs reported in $\geq 30 \%$ of patients in the safety population in either treatment group are shown in Fig. 1. The most common TEAEs with cabozantinib included fatigue, hypertension, diarrhoea, abnormal liver function test, anorexia and palmar-plantar erythrodysesthesia syndrome (PPES) (Fig. 1) [21]. Sunitinib had a similar overall tolerability profile, with lower incidences of PPES, weight loss and anorexia, but higher incidences of haematological toxicities (i.e. neutropenia and thrombocytopenia) (Fig. 1). Grade 3-4 hypertension, diarrhoea, fatigue and oral mucositis were reported in $\geq 5 \%$ of patients in both treatment groups. Other grade 3-4 TEAEs reported in $\geq 5 \%$ patients were PPES, anorexia and increased ALT levels in cabozantinib recipients, and thrombocytopenia in sunitinib recipients (Fig. 1) [21]. Grade 5 TRAEs occurred in three cabozantinib recipients (once case each of acute kidney injury, sepsis and jejunal perforation) and three sunitinib recipients (one case each of sepsis, respiratory failure and vascular disorders) [21].

Potentially serious adverse events may occur with the use of cabozantinib [27]. Patients should be closely evaluated for TRAEs during the first 8 weeks of treatment, as most occur early during the course of treatment and dosage reductions, treatment interruption and/or treatment discontinuation may be needed [7, 27]. In CABOSUN [21], 46\% of cabozantinib recipients required dosage reductions, and $20 \%$ discontinued treatment because of TEAEs; the corresponding proportions in sunitinib recipients are 35 and $21 \%$ [21]. Table 3 summaries the incidence and recommended management of potentially serious TRAEs, and Fig. 2 provides an overall algorithm for the management of grade 1-4 TRAEs based on their severity $[7,8,27]$.

The tolerability of cabozantinib relative to first-line treatments other than sunitinib has not been directly compared. In the NMA that evaluated tolerability of cabozantinib versus other first-line options, there was no difference between cabozantinib and sunitinib with regard to the risk of TEAEs (odds ratio 1.1; 95\% CI 0.59-2.2), but cabozantinib had an increased risk relative to nivolumab + ipilimumab $(2.2 ; 1.1-4.4)$, with the latter having a $67 \%$ likelihood of being the best tolerated regimen for the first-line systemic treatment of mRCC [28].

Data related to the tolerability of cabozantinib in the first-line treatment of aRCC in the real-world setting are currently not available. Real-world tolerability data, however, are available from 96 patients with $\mathrm{mRCC}$ who received second- or subsequent line treatment with cabozantinib within the Italian Managed Access Program (MAP) [29]. This analysis indicated that the tolerability of cabozantinib in the real-world setting was manageable and comparable to that shown in the RCC clinical trial program [29]. Of note, in an analysis of 22 patients with mRCC in a single Italian MAP centre [30], 6 months of treatment with cabozantinib appeared to significantly

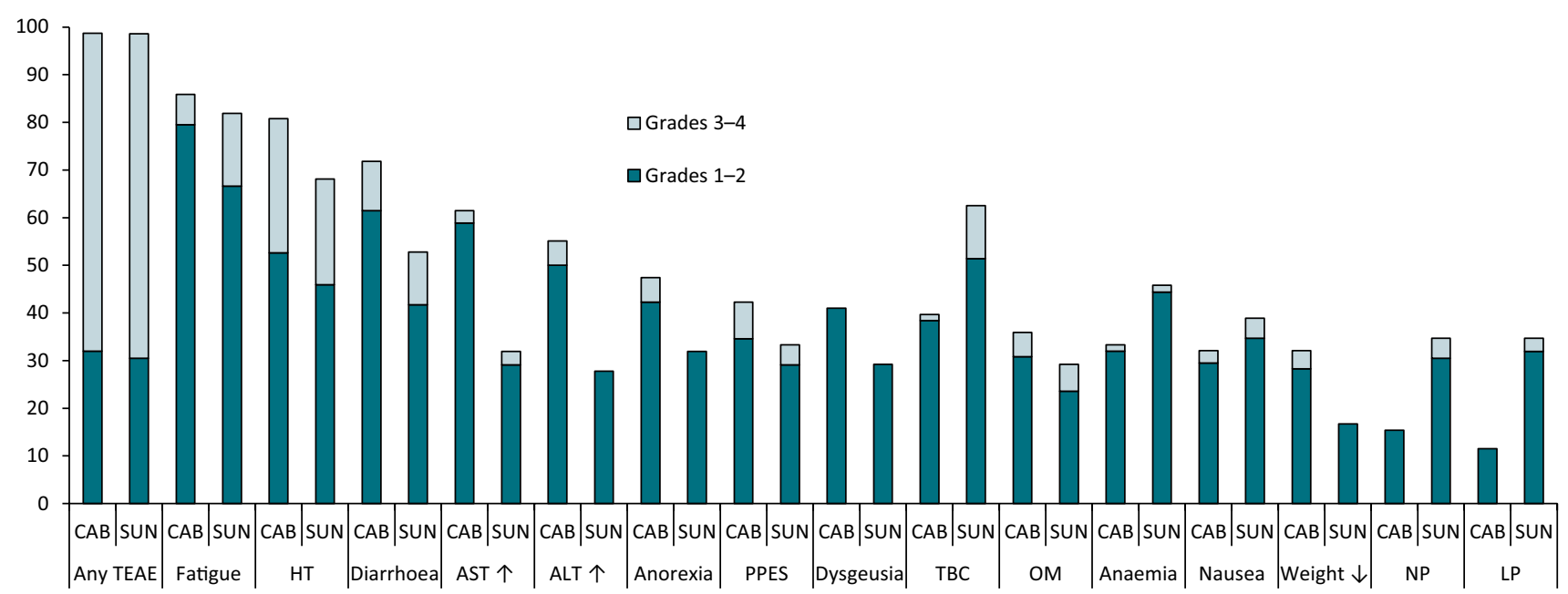

Fig. 1 Incidence of grade 1-2 and grade 3-4 treatment-emergent adverse events (TEAEs) reported in $\geq 30 \%$ of patients in the safety population of either the cabozantinib $(\mathrm{CAB} ; n=78)$ or sunitinib (SUN; $n=72$ ) treatment group in CABOSUN [21]. TEAE severity was graded according to National Cancer Institute Common Termi- nology Criteria for Adverse Events version 4.0. $H T$ hypertension, $L P$ leukopenia, $N P$ neutropenia, $O M$ oral mucositis, $P P E S$ palmar-plantar erythrodysesthesia syndrome, $T B C$ thrombocytopenia, $\uparrow$ increase, $\downarrow$ decrease 
$(p<0.05)$ reduce bone resorption from baseline; further evaluation of this effect in a larger population would be of interest.

Treatment with cabozantinib $60 \mathrm{mg} /$ day was not associated with any incidents of prolongation of the corrected QT (QTc) interval in patients with the CABOSUN trial [31]. In a phase 3 trial in patients with medullary thyroid cancer, cabozantinib at the higher dosage of $140 \mathrm{mg} /$ day increased the $\Delta-\Delta$ Fridericia QTc interval by $10-15 \mathrm{~ms}$, with this prolongation thought to be due to the effects of cabozantinib on serum calcium and potassium [31]. In the EU, caution is required when using cabozantinib in patients with a history of QT interval prolongation or relevant pre-existing cardiac disease, bradycardia or electrolyte disturbances, or who are taking antiarrhythmics [7].

\section{What is the current clinical position of cabozantinib as first-line treatment of aRCC?}

Cabozantinib is an important guideline-recommended firstline option for the treatment of aRCC due to its:

- Mechanism of action Has anti-angiogenic properties and potently inhibits multiple tyrosine kinases implicated in the development of RCC [3, 4, 7, 8, 12-15].

- Overall efficacy In patients with mRCC of IMDC poor or intermediate risk, targeted first-line treatment with cabozantinib significantly prolonged the primary endopoint of median PFS (investigator and independent assessments), and increased the ORR (investigator assessment) relative to standard-of-care sunitinib [21, 22].

- Manageable tolerability profile TRAEs are manageable with supportive care, dosage reductions and/or treatment interruption or permanent discontinuation $[7,8]$.

Table 3 Incidence and management of potentially serious adverse effects associated with the use of oral cabozantinib tablets (Cabometyx $\left.{ }^{\circledR}\right)$ in clinical trials in patients with renal cell carcinoma [7,8]. Unless otherwise indicated, information applies to both the EU and USA

Adverse effect (incidence in RCC trials [8]) Prevention/management [7, 8]

Diarrhoea (74\%, including $11 \%$ with grade 3) Intolerable grade 2 or grade 3-4 diarrhoea that cannot be managed with standard antidiarrhoeal treatments: withhold cabozantinib until improvement to grade 1 ; resume cabozantinib at a $\downarrow$ dosage $^{\text {a }}$

Fistulas (1\%) and gastrointestinal perforations $(1 \%)$

Haemorrhage (3\% with grade $\geq 3$ )

Hypertension (44\%, including $18 \%$ with grade $\geq 3$ )

Nephrotic syndrome

PPES (42\%, including $8 \%$ with grade 3)

RPLS

Thrombotic events ( 9 and $1 \%$ with venous and arterial thromboembolism, respectively)

Wound complications and surgery
Monitor pts for symptoms, including abscess and sepsis

Fistula or gastrointestinal perforation that cannot be appropriately managed: permanently discontinue cabozantinib

Pts with, or who are at risk for, severe haemorrhage: do not use cabozantinib

Severe haemorrhage: permanently discontinue cabozantinib

Monitor BP before and during cabozantinib treatment; control BP before starting cabozantinib

Inadequately controlled BP despite medical management: withhold cabozantinib; resume cabozantinib at a $\downarrow$ dosage ${ }^{\mathrm{a}}$ once BP is controlled

Hypertensive crisis or severe uncontrolled BP despite optimal medical management: permanently discontinue cabozantinib

Monitor urine protein regularly (EU)

Development of nephrotic syndrome: permanently discontinue cabozantinib

Intolerable grade 2 or grade 3 PPES: withhold cabozantinib until improvement to grade 1; resume cabozantinib at a $\downarrow$ dosage ${ }^{\text {a }}$

Pt presents with seizures, headache, visual disturbances, confusion or altered mental function: evaluate for RPLS

Development of RPLS: permanently discontinue cabozantinib

Development of acute myocardial infarction or any other clinically significant arterial thromboembolic complication: permanently discontinue cabozantinib

Pts who are at risk for, or have a history of, venous or arterial thromboembolism: use cabozantinib with caution (EU)

Scheduled surgery (including dental): stop cabozantinib $\geq 28$ days before surgery if possible

Resume cabozantinib once the wound is adequately healed (EU)

Inadequate wound healing required medical intervention: discontinue cabozantinib (EU)

Toxicity severity was graded according to National Cancer Institute Common Terminology Criteria for Adverse Events version 4.0

$B P$ blood pressure, PPES palmar-plantar erythrodysesthesia syndrome, $p t$ patient, RCC renal cell carcinoma, RPLS reversible posterior leukoenecephalopathy syndrome, $\downarrow$ reduced

${ }^{\mathrm{a}}$ From $60 \mathrm{mg} /$ day to $40 \mathrm{mg} /$ day, and from $40 \mathrm{mg} /$ day to $20 \mathrm{mg} /$ day 


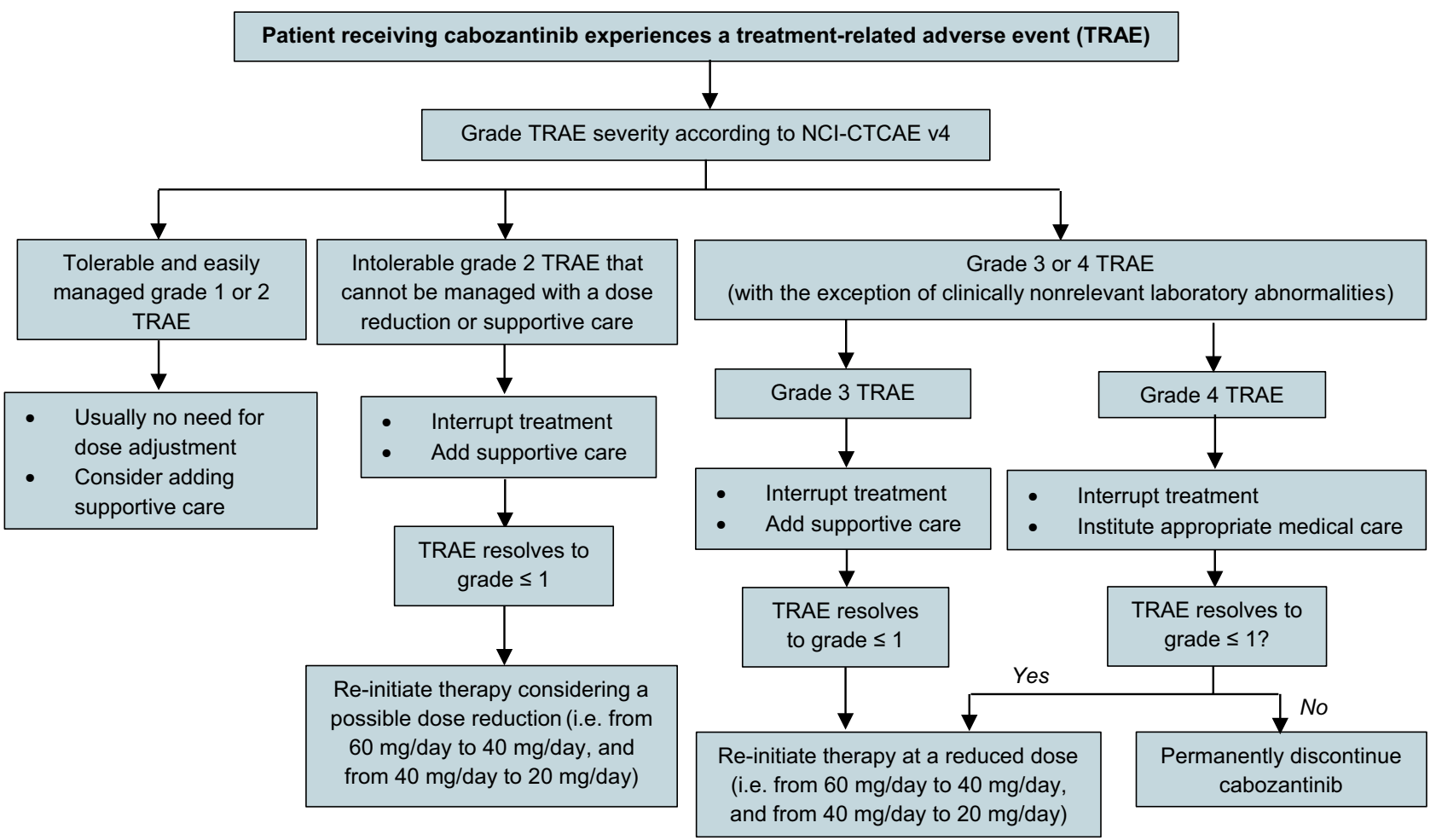

Fig. 2 Recommended dose modifications for cabozantinib based on toxicity severity [graded according to National Cancer Institute Common Terminology Criteria for Adverse Events version 4.0 (NCI-CTCAE v4)] [7, 8]

In the setting of the treatment of IMDC intermediateand poor-risk clear-cell aRCC/mRCC in treatment-naïve patients, cabozantinib has been included in the following recently updated guidelines:

- Updated European Association of Urology (EAU) guidelines for the treatment of first-line metastatic clear cell renal cancer [6] Options for the first-line treatment of IMDC intermediate-and poor-risk clear-cell aRCC/ mRCC include nivolumab + ipilimumab (strong recommendation based on the clinical evidence), and cabozantinib and sunitinib (weak recommendations based on current clinical evidence). Pazopanib is recommended for intermediate-risk patients only (weak recommendation).

- National Comprehensive Cancer Network ${ }^{\circledR}$ (NCCN Guidelines $^{\circledR}$ ): kidney cancer (version 4.2018) [1] In alphabetical order by category and preference, options for the first-line treatment of predominantly clear-cell renal carcinoma of IMDC intermediate and poor risk include nivolumab + ipilimumab (NCCN category 1, preferred option in these risk groups), pazopanib, sunitinib and bevacizumab + IFN $\alpha-2 B$ (category 1), temsirolimus (category 1 for poor risk and $2 \mathrm{~B}$ for selected patients in other risk groups), axitinib and cabozantinib (both category 2A), and best supportive care (e.g. palliative radiotherapy, metastasectomy, ablative therapy, bisphosphonates, etc.). NCCN categories 1 and 2A are defined as uniform NCCN consensus that that the intervention is appropriate based on high- and lower-level evidence, respectively, and category $2 \mathrm{~B}$ as $\mathrm{NCCN}$ consensus that that the intervention is appropriate based on lower-level evidence [1].

Acknowledgements The manuscript was reviewed by: E. Gallardo, Medical Oncology Department, Parc Taulí Hospital Universitari, Institut d'Investigació i Innovació ParcTaulí I3P, Universitat Autònoma de Barcelona, Sabadell, Spain; B. Maughan, Department of Oncology, Huntsman Cancer Institute, University of Utah, Salt Lake City, UT, USA. During the peer review process, Ipsen Pharma (EU) and Exelixis, Inc. (USA), the marketing-authorization holders of cabozantinib, were also offered an opportunity to provide a scientific accuracy review of their data. Changes resulting from comments received were made on the basis of scientific and editorial merit.

\section{Compliance with ethical standards}

Funding The preparation of this review was not supported by any external funding. 
Conflicts of interest K. A. Lyseng-Williamson is an employee of Adis/ Springer, is responsible for the article content and declares no conflicts of interest.

Open Access This article is distributed under the terms of the Creative Commons Attribution-NonCommercial 4.0 International License (http://creativecommons.org/licenses/by-nc/4.0/), which permits any noncommercial use, duplication, adaptation, distribution and reproduction in any medium or format, as long as you give appropriate credit to the original author(s) and the source, provide a link to the Creative Commons license and indicate if changes were made.

\section{References}

1. National Comprehensive Cancer Network ${ }^{\circledR}$. Clinical Practice Guidelines in Oncology (NCCN Guidelines $\left.{ }^{\circledR}\right)$ : kidney cancer (version 4.2018). Fort Washington: National Comprehensive Cancer Network ${ }^{\circledR}$, Inc.; 2018.

2. Garcia-Roig M, Ortiz N, Lokeshwar V. Molecular marker for predicting treatment response in advanced renal cell carcinoma: does the promise fulfill clinical need? Curr Urol Rep. 2014;15:375.

3. Tannir NM, Schwab G, Grunwald V. Cabozantinib: an active novel multikinase inhibitor in renal cell carcinoma. Curr Oncol Rep. 2017;19(2):14

4. Yu SS, Quinn DI, Dorff TB. Clinical use of cabozantinib in the treatment of advanced kidney cancer: efficacy, safety, and patient selection. Onco Targets Ther. 2016;9:5825-37.

5. Gill DM, Hahn AW, Hale P, et al. Overview of current and future first-line systemic therapy for metastatic clear cell renal cell carcinoma. Curr Treat Options Oncol. 2018;19(1):6.

6. Powles T, Albiges L, Staehler M, et al. Updated European Association of Urology Guidelines recommendations for the treatment of first-line metastatic clear cell renal cancer. Eur Urol. 2018;73(3):311-5.

7. Cabometyx ${ }^{\circledR}$ (cabozantinib) 20,40 and $60 \mathrm{mg}$ oral tablets: summary of product characteristics. London: European Medicines Agency; 2018.

8. Cabometyx ${ }^{\circledR}$ (cabozantinib) 20,40 and $60 \mathrm{mg}$ tablets, for oral use: US prescribing Information. South San Francisco: Exelixis, Inc.; 2017

9. Cometriq ${ }^{\circledR}$ (cabozantinib) 20 and $80 \mathrm{mg}$ capsules: summary of product characteristics. London: European Medicines Agency; 2018.

10. Cometriq ${ }^{\circledR}$ (cabozantinib) 20 and $80 \mathrm{mg}$ capsules, for oral use: US prescribing Information. South San Francisco: Exelixis, Inc.; 2018.

11. Nguyen L, Benrimoh N, Xie Y, et al. Pharmacokinetics of cabozantinib tablet and capsule formulations in healthy adults. Anticancer Drugs. 2016;27(7):669-78.

12. Yakes FM, Chen J, Tan J, et al. Cabozantinib (XL184), a novel MET and VEGFR2 inhibitor, simultaneously suppresses metastasis, angiogenesis, and tumor growth. Mol Cancer Ther. 2011;10(12):2298-308.

13. Xie Z, Lee YH, Boeke M, et al. MET inhibition in clear cell renal cell carcinoma. J Cancer. 2016;7(10):1205-14.

14. Zhou L, Liu XD, Sun M, et al. Targeting MET and AXL overcomes resistance to sunitinib therapy in renal cell carcinoma. Oncogene. 2016;35(21):2687-97.

15. Lacy SA, Miles DR, Nguyen LT. Clinical pharmacokinetics and pharmacodynamics of cabozantinib. Clin Pharmacokinet. 2017;56(5):477-91

16. Lacy S, Nielsen J, Yang B, et al. Population exposure-response analysis of cabozantinib efficacy and safety endpoints in patients with renal cell carcinoma. Cancer Chemother Pharmacol. 2018;81(6):1061-70.
17. Nguyen L, Holland J, Ramies D, et al. Effect of renal and hepatic impairment on the pharmacokinetics of cabozantinib. J Clin Pharmacol. 2016;56(9):1130-40.

18. Lacy SA, Hsu B, Miles D, et al. Metabolism and disposition of cabozantinib in healthy male volunteers and pharmacologic characterization of its major metabolites. Drug Metab Dispos. 2015;43(8):1190-207.

19. Nguyen L, Holland J, Mamelok R, et al. Evaluation of the effect of food and gastric $\mathrm{pH}$ on the single-dose pharmacokinetics of cabozantinib in healthy adult subjects. J Clin Pharmacol. 2015;55(11):1293-301.

20. Nguyen L, Holland J, Miles D, et al. Pharmacokinetic (PK) drug interaction studies of cabozantinib: effect of CYP3A inducer rifampin and inhibitor ketoconazole on cabozantinib plasma PK and effect of cabozantinib on CYP2C8 probe substrate rosiglitazone plasma PK. J Clin Pharmacol. 2015;55(9):1012-23.

21. Choueiri TK, Halabi S, Sanford BL, et al. Cabozantinib versus sunitinib as initial targeted therapy for patients with metastatic renal cell carcinoma of poor or intermediate risk: the Alliance A031203 CABOSUN trial. J Clin Oncol. 2017;35(6):591-7 [erratum J Clin Oncol. 2017;35(32):3736].

22. Choueiri TK, Hessel C, Halabi S, et al. Cabozantinib versus sunitinib as initial therapy for metastatic renal cell carcinoma of intermediate or poor risk (Alliance A031203 CABOSUN randomised trial): progression-free survival by independent review and overall survival update. Eur J Cancer. 2018;94:115-25.

23. Feldman DR, Feuilly M, Meng J, et al. ECOG score analysis as a proxy for health-related quality of life assessment in patients with poor or intermediate risk metastatic renal cell carcinoma from the CABOSUN trial (Alliance A031203) [abstract no. e18690]. J Clin Oncol. 2018;36(Suppl).

24. Chen RC, Feuilly M, Meng J, et al. Quality-adjusted time without symptoms or toxicity (Q-TWiST): analysis of cabozantinib (Cabo) vs sunitinib (Sun) in patients with advanced renal cell carcinoma (aRCC) of intermediate or poor risk (Alliance A031203) [abstract no. 4556]. J Clin Oncol. 2018;36(Suppl).

25. Schmidt E, Lister J, Neumann M, et al. Cabozantinib versus standard-of-care comparators in the treatment of advanced/ metastatic renal cell carcinoma in treatment-naive patients: a systematic review and network meta-analysis. Target Oncol. 2018;13(2):205-16.

26. Wallis CJD, Klaassen Z, Bhindi B, et al. First-line systemic therapy for metastatic renal cell carcinoma: a systematic review and network meta-analyses. Eur Urol. 2018. https://doi.org/10.1016/j. eururo.2018.03.036 (Epub 2018).

27. Schmidinger M, Danesi R. Management of adverse events associated with cabozantinib therapy in renal cell carcinoma. Oncologist. 2018;23(3):306-15.

28. George DJ, Hessel C, Halabi S, et al. Cabozantinib versus sunitinib for previously untreated patients with advanced renal cell carcinoma (RCC) of intermediate or poor risk: subgroup analysis of progression-free survival (PFS) and objective response rate (ORR) in the Alliance A031203 CABOSUN trial [abstract no. 582]. J Clin Oncol. 2018;36(Suppl 6S).

29. Procopio G, Prisciandaro M, Iacovelli R, et al. Safety and efficacy of cabozantinib in metastatic renal-cell carcinoma: real-world data from an Italian managed access program. Clin Genitourin Cancer. 2018;16(4):e945-e951.

30. Ratta R, Verzoni E, Pantano F, et al. Effects of cabozantinib on bone turnover markers in patients with metastatic renal cell carcinoma [abstract no. 638]. J Clin Oncol. 2018;36(Suppl 6S).

31. Miles DR, Lacy SA, Wada DR, et al. Assessment of cabozantinib treatment on QT interval in a phase 3 study in medullary thyroid cancer: evaluation of indirect QT effects mediated through treatment-induced changes in serum electrolytes. Cancer Chemother Pharmacol. 2017;80(2):295-306. 